PROCEEDINGS OF THE

AMERICAN MATHEMATICAL SOCIETY

Volume 140, Number 9, September 2012, Pages 3151-3160

S 0002-9939(2012)11260-2

Article electronically published on January 6, 2012

\title{
LINEAR ORTHOGONALITY PRESERVERS OF HILBERT $C^{*}$-MODULES OVER $C^{*}$-ALGEBRAS WITH REAL RANK ZERO
}

\author{
CHI-WAI LEUNG, CHI-KEUNG NG, AND NGAI-CHING WONG
}

(Communicated by Marius Junge)

\begin{abstract}
Let $A$ be a $C^{*}$-algebra with real rank zero. Let $E$ and $F$ be Hilbert $A$-modules with $E$ being full. Suppose that $\theta: E \rightarrow F$ is a linear map preserving orthogonality, i.e.,

$$
\langle\theta(x), \theta(y)\rangle=0 \quad \text { whenever }\langle x, y\rangle=0 .
$$

We show in this article that if $\theta$ is an $A$-module map (not assumed to be bounded), then there exists a central positive multiplier $u \in M(A)$ such that

$$
\langle\theta(x), \theta(y)\rangle=u\langle x, y\rangle \quad(x, y \in E) .
$$

In the case when $A$ is a standard $C^{*}$-algebra, when $A$ is a real rank zero properly infinite unital $C^{*}$-algebra, or when $A$ is a $W^{*}$-algebra, we also get the same conclusion with the assumption of $\theta$ being an $A$-module map weakened to being a local map.
\end{abstract}

\section{InTRODUCTION AND NOTATION}

It is common knowledge that, together with linearity, the inner product and the norm structures of a Hilbert space $H$ determine each other. It might be a bit less well known that the orthogonality structure also suffices to determine the inner product up to a scalar. This fact follows from the following easy observation: for any $x, y \in H,\|x\|=\|y\|$ if and only if $x+\lambda y$ is orthogonal to $x-$ $\lambda y$ for some scalar $\lambda$ with $|\lambda|=1$ (see also [3, 6]).

It is natural and interesting to ask whether the linear structure and orthogonality structure of a (complex) Hilbert $C^{*}$-module determines its $C^{*}$-algebra-valued inner product. More precisely, let $A$ be a (complex) $C^{*}$-algebra, and $\theta: E \rightarrow F$ be a $\mathbb{C}$ linear map between Hilbert $A$-modules that preserves orthogonality (i.e. preserves zero $A$-valued inner products). We want to study to what extent $\theta$ will respect the $A$-valued inner products. When the underlying $C^{*}$-algebra is $\mathbb{C}$, it reduces to the case of Hilbert spaces.

We first note that without any further assumption on $\theta$, the above question might have a negative answer.

Received by the editors May 25, 2010 and, in revised form, March 22, 2011.

2010 Mathematics Subject Classification. Primary 46L08, 46H40.

Key words and phrases. Hilbert $C^{*}$-modules, orthogonality preservers, module maps, local maps, real rank zero $C^{*}$-algebras.

The authors are supported by The Chinese University of Hong Kong Direct Grant (2060389), National Natural Science Foundation of China (10771106), and Taiwan NSC grant (NSC96-2115M-110-004-MY3). 
Example 1.1. Let $H$ be an infinite dimensional (complex) Hilbert space and $A=$ $\mathcal{K}(H)$ be the $C^{*}$-algebra of all compact operators on $H$. Suppose that $\bar{H}$ is a vector space that is conjugate-linear isomorphic to $H$. When equipped with the operations: $\left\langle\overline{\eta_{1}}, \overline{\eta_{2}}\right\rangle:=\eta_{1} \otimes \eta_{2}$ and $\overline{\eta_{1}} T:=\overline{T^{*} \eta_{1}}\left(\overline{\eta_{1}}, \overline{\eta_{2}} \in \bar{H} ; T \in A\right)$, we see that $\bar{H}$ is a Hilbert $A$-module. Suppose that $\theta$ is any unbounded bijective $\mathbb{C}$-linear map from $\bar{H}$ onto $\bar{H}$. Since $\langle x, y\rangle=0$ if and only if $x=0$ or $y=0$, we see that both $\theta$ and $\theta^{-1}$ preserve orthogonality.

In fact, assuming norm continuity, Schweizer obtains his pioneering work in his thesis (see [20,9.6]) on a characterization of bounded orthogonality-preserving $\mathbb{C}$-linear maps. His result ensures that all bounded orthogonality-preserving $\mathbb{C}$ linear maps from a full Hilbert $C$-module $X$ into a full Hilbert $D$-module $Y$ are exactly those maps $T$ which induce a sort of "correspondence" between the $C^{*}$ algebras $\mathcal{K}(X)$ and $\mathcal{K}(Y)$, where $\mathcal{K}(X)$ (respectively, $\mathcal{K}(Y)$ ) is generated by "rank one operators" $\theta_{\zeta, \eta}$ defined as $\theta_{\zeta, \eta}(\xi):=\zeta\langle\eta, \xi\rangle$ for $\zeta, \eta, \xi \in X$ (respectively, $Y$ ). More precisely, there is a "local morphism" $\pi: \mathcal{K}(X) \rightarrow \mathcal{K}(Y)$ such that

$$
T(a x)=\pi(a) T(x) \quad(a \in \mathcal{K}(X)),
$$

or equivalently,

$$
\theta_{T \zeta, T \zeta} \leq\|T\|^{2} \pi\left(\theta_{\zeta, \zeta}\right) \quad(\zeta \in X) .
$$

As a result, in the case $C=D$, we can see that even a bounded $\mathbb{C}$-linear orthogonality preserver does not necessarily preserve the $C^{*}$-algebra inner products. Hence, we still need more assumptions to ensure our question to have a positive answer.

As we are dealing with Hilbert $A$-modules, a natural additional assumption is that $\theta$ is an $A$-module map, i.e., $\theta(x a)=\theta(x) a(x \in E, a \in A)$. In [9], Ilišević and Turnšek showed that if $A$ is a standard $C^{*}$-algebra, then for every orthogonalitypreserving $A$-module map $\theta: E \rightarrow F$, there is a scalar $\lambda \geq 0$ such that

$$
\langle\theta(x), \theta(y)\rangle=\lambda\langle x, y\rangle \quad(x, y \in E) .
$$

In particular, all such $\theta$ are scalar multiples of isometries.

In [13, under a weaker assumption on $\theta$, namely $\theta$ being "local", we get a similar conclusion in the case when $A$ is a commutative $C^{*}$-algebra (in fact, the main difficulties in 13 come from the fact that $\theta$ is not assumed to be an $A$-module map). Recall that a $\mathbb{C}$-linear map $\theta: E \rightarrow F$ is local if

$$
\theta(x) a=0 \quad \text { whenever } \quad x a=0 \quad(x \in E ; a \in A) .
$$

Readers should find the idea of local linear maps familiar. For example, local linear maps on the space of smooth functions defined on a manifold modeled on $\mathbb{R}^{n}$ are exactly linear differential operators (see, e.g., [19, 16]). See [11, 2] for the vectorvalued case, and 1 for the Banach $C^{1}[0,1]$-module setting. We also mention that there is a bimodule version of local maps as studied by Schweizer in 20, 21] (which is different from ours). Notice that every module map is local, but local linear maps, e.g., linear differential operators, might not be a module map. Nevertheless, it has been shown in [12, Proposition A.1] that every bounded local map between Hilbert $C^{*}$-modules is a module map.

The results in [9] and [13] lead to the following conjecture. We remark that the fullness assumption of $E$ in this conjecture is a necessity. Without this, the conclusion does not hold even in the case when $A$ is commutative (see [13, 3.6]). Here, a Hilbert $A$-module $E$ is said to be full if the linear span of $\{\langle x, y\rangle: x, y \in E\}$ is dense in $A$. 
Conjecture 1.2. Let $A$ be $a C^{*}$-algebra. Let $E$ and $F$ be Hilbert $A$-modules with $E$ being full. Assume $\theta: E \rightarrow F$ is a (C-linear) local map preserving orthogonality; i.e. for any $x, y \in E$,

$$
\langle x, y\rangle=0 \quad \text { implies }\langle\theta(x), \theta(y)\rangle=0 .
$$

Then there is a central positive element $u \in M(A)$ such that

$$
\langle\theta(x), \theta(y)\rangle=u\langle x, y\rangle \quad(x, y \in E) .
$$

In this article, positive answers of this conjecture will be given in the following four cases:

(1) $A$ is a $C^{*}$-algebra of real rank zero and $\theta$ is an $A$-module map (Theorem 2.3).

(2) $A$ is a standard $C^{*}$-algebra (Corollary [3.2).

(3) $A$ is a properly infinite unital $C^{*}$-algebra of real rank zero (Corollary 3.3).

(4) $A$ is a $W^{*}$-algebra (Corollary 3.4).

We note that although the main result, Theorem 9.6, in [20] appears to be more general and more complete (as they deal with $\mathbb{C}$-linear orthogonality preservers), they do not give too much hint to the answer of our conjecture. For example, in the situation of Example 1.1, what one can get from [20, 9.6] is the tautology that a bounded orthogonality-preserving $\mathbb{C}$-linear map $\theta: \bar{H} \rightarrow \bar{H}$ is $\mathbb{C}$-linear. This does not give any useful insight into the study of orthogonality-preserving $\mathcal{K}(H)$-module maps in even such a simple case.

As a final remark for the introduction, we note that, unlike the situation in some of the other literature (e.g. [20, 7]), $\theta$ is not assumed to be bounded. It is because of the conceptual reason that $A$-linearity (or locality) and orthogonality structures should completely determine the $A$-inner products, as stated in the beginning of this section. However, we shall see that the boundedness of an $A$-linear orthogonality preserver will be an automatic consequence of our results.

\section{Orthogonality-Preserving $A$-module maps}

First, we give some notation that will be used throughout this article. In the following, $A$ is a $C^{*}$-algebra, $E$ and $F$ are Hilbert $A$-modules, and $\Psi, \theta: E \rightarrow F$ are orthogonality-preserving $\mathbb{C}$-linear maps, which are not assumed to be bounded.

Let $a \in A_{+}$. We set $C^{*}(a)$ to be the $C^{*}$-subalgebra generated by $a$, and $\mathbf{c}(a)$ to be the central cover of $a$, i.e., the smallest central element in $A_{+}^{* *}$ dominating $a$ (see, e.g., [18, 2.6.2]). If, in addition, $\alpha, \beta \in \mathbb{R}_{+}$, we put $e_{a}(\alpha, \beta]$ to be the spectral projections of $a$ in $A^{* *}$ corresponding to the set $(\alpha, \beta] \cap \sigma(a)$.

We denote by $Z(A)$ the center and by $M(A)$ the space of all multipliers of $A$. On the other hand, $\operatorname{Proj}_{1}(A)$ is the set of all norm-one (i.e., nonzero) projections in $A$. For any open projection $p \in \operatorname{Proj}_{1}\left(A^{* *}\right)$ (i.e., there exists an increasing net $\left\{a_{i}\right\}$ in $A_{+}$such that $a_{i} \uparrow p$ in the weak-*-topology), we denote by $\operatorname{her}(p):=p A^{* *} p \cap A$ the hereditary $C^{*}$-subalgebra associated to $p$. See, e.g., 4] for more information about open projections.

We recall that $A$ has real rank zero if every selfadjoint element in $A$ can be approximated in norm by invertible selfadjoint elements. If $A$ has real rank zero, then the real linear span of $\operatorname{Proj}_{1}(A)$ is norm dense in $A_{s a}$ (see, e.g., [5]). 
Let us start with the following easy lemma, part (a) of which might be well known, but we give an argument here for completeness.

Lemma 2.1. (a) If $p \in \operatorname{Proj}_{1}\left(A^{* *}\right)$ and $b \in Z\left(p A^{* *} p\right)_{+}$, then $\|\mathbf{c}(b)\|=\|b\|, \mathbf{c}(b) p=$ $b$ and $\mathbf{c}(b) \mathbf{c}(p)=\mathbf{c}(b)$.

(b) Suppose that $A$ has real rank zero and $E$ is full. If $q \in A^{* *} \backslash\{0\}$ is an open projection, there are $r \in \operatorname{Proj}_{1}(A)$ and $y \in E r$ such that $r=\langle y, y\rangle \leq q$.

Proof. (a) Since $b \leq\|b\| 1$, we see that $0 \leq b \leq \mathbf{c}(b) \leq\|b\| 1$ and $\|\mathbf{c}(b)\|=\|b\|$. Clearly, $\mathbf{c}(b) p=p \mathbf{c}(b) p \geq p b p=b$. Conversely, as $Z\left(p A^{* *} p\right)=Z\left(A^{* *}\right) p$ (see e.g. [10, 5.5.6]), there is $a \in Z\left(A^{* *}\right)_{+}$with $b=a p$ (note that $\left.b^{1 / 2} \in Z\left(A^{* *}\right) p\right)$. Thus, we have $b=a^{1 / 2} p a^{1 / 2} \leq a^{1 / 2} \mathbf{c}(p) a^{1 / 2}=a \mathbf{c}(p)$. As $a \mathbf{c}(p)$ is central, $\mathbf{c}(b) \leq a \mathbf{c}(p)$ and $\mathbf{c}(b) p=p \mathbf{c}(b) p \leq a p=b$. The last equality follows from [18, 2.6.4] and the fact that $b \mathbf{c}(p)=b$.

(b) Note that her $(q) \neq(0)$ and also has real rank zero (see e.g. [5]). Moreover, $E_{0}:=E \cdot h e r(q)$ is a full (and, hence nonzero) Hilbert her $(q)$-module. Pick any $x \in E_{0}$ such that $a:=\langle x, x\rangle$ is a norm-one element in her $(q)$. Let $t \in(0,1 / 3)$ and $b \in \operatorname{her}(q)_{+}$such that $\|a-b\|<t$ and $\sigma(b)=\left\{\lambda_{1}, \ldots, \lambda_{n}\right\}$ with $\lambda_{1} \leq \cdots \leq$ $\lambda_{n}=\|b\|$ (see e.g. [5]). Since $\|b\|>2 / 3$, we can choose $s \in[t,\|b\|] \backslash \sigma(b)$. If we set $r:=e_{b}(s, 2] \in \operatorname{Proj}_{1}(A)$, then $\|r-r a r\| \leq\|r-r b r\|+\|b-a\|<1$. If $c:=r+\sum_{n=1}^{\infty}(r-\operatorname{rar})^{n} \in A_{+}$, then $($rar $) c=c($ rar $)=r$ and so, $\left\langle x c^{1 / 2}, x c^{1 / 2}\right\rangle=$ $c^{1 / 2} \operatorname{rarc}^{1 / 2}=r$. Finally, $x c^{1 / 2} \in E r$ as $c^{1 / 2}$ is in the $C^{*}$-subalgebra $r A r+\mathbb{C r}$.

Proposition 2.2. Let $A$ be a unital $C^{*}$-algebra of real rank zero. Suppose that $\theta: E \rightarrow F$ is an A-module map preserving orthogonality, and there is an element $x_{0} \in E$ such that $\left\langle x_{0}, x_{0}\right\rangle=1$. Then one can find $u \in Z(A)_{+}$satisfying

$$
\langle\theta(x), \theta(y)\rangle=u\langle x, y\rangle \quad(x, y \in E) .
$$

Proof. Let $u:=\left\langle\theta\left(x_{0}\right), \theta\left(x_{0}\right)\right\rangle \in A_{+}$. For any symmetry $w \in A$, as $x_{0}+x_{0} w$ and $x_{0}-x_{0} w$ are orthogonal to each other, so are $\theta\left(x_{0}\right)+\theta\left(x_{0}\right) w$ and $\theta\left(x_{0}\right)-\theta\left(x_{0}\right) w$. Consequently, $u+w u-u w-w u w=0$ and $u+u w-w u-w u w=0$ (by taking the adjoint). This tells us that $u=w u w$, and so $u \in Z(A)_{+}$(as $A$ is generated by projections, and thus also by symmetries). Pick any $z \in E$ with $\left\langle x_{0}, z\right\rangle=0$. Then $z+x_{0}\langle z, z\rangle^{1 / 2}$ is also orthogonal to $z-x_{0}\langle z, z\rangle^{1 / 2}$. It follows from the orthogonalitypreserving property that

$$
\langle\theta(z), \theta(z)\rangle=\langle z, z\rangle^{1 / 2}\left\langle\theta\left(x_{0}\right), \theta\left(x_{0}\right)\right\rangle\langle z, z\rangle^{1 / 2}=u\langle z, z\rangle .
$$

For any $y \in E$, the element $z=y-x_{0}\left\langle x_{0}, y\right\rangle$ is orthogonal to $x_{0}$. Hence,

$$
\langle\theta(y), \theta(y)\rangle=\left\langle y, x_{0}\right\rangle\left\langle\theta\left(x_{0}\right), \theta\left(x_{0}\right)\right\rangle\left\langle x_{0}, y\right\rangle+\langle\theta(z), \theta(z)\rangle=u\langle y, y\rangle .
$$

A polarization type argument implies that $\langle\theta(x), \theta(y)\rangle=u\langle x, y\rangle(x, y \in E)$.

Theorem 2.3. Let $A$ be a $C^{*}$-algebra of real rank zero. Suppose that $E$ is full, and $\theta: E \rightarrow F$ is an orthogonality-preserving A-module map (not assumed to be bounded). There is $u \in Z(M(A))_{+}$such that

$$
\langle\theta(x), \theta(y)\rangle=u\langle x, y\rangle \quad(x, y \in E) .
$$

In particular, $\theta$ is automatically bounded.

Proof. Set

$$
P:=\left\{(x, p) \in E \times \operatorname{Proj}_{1}(A):\langle x, x\rangle=p\right\} .
$$


Lemma 2.1(b) tells us that $P \neq \emptyset$. Suppose that $(x, p) \in P$. Then $x p=x$ since we have $\langle x p-x, x p-x\rangle=p\langle x, x\rangle p-\langle x, x\rangle p-p\langle x, x\rangle+\langle x, x\rangle=0$. Also, $E p$ is a full Hilbert $p A p$-module and the restriction of $\theta$ on $E p$ is an orthogonality-preserving $p A p$-module map. Since $p$ is the identity of the $C^{*}$-algebra $p A p$ and $\theta(E p) \subseteq F p$, one can apply Proposition 2.2 to obtain $b_{p} \in Z(p A p)_{+}$which satisfies

$$
\langle\theta(x) p, \theta(y) p\rangle=b_{p}\langle x p, y p\rangle \quad(x, y \in E) .
$$

By Lemma 2.1(a), we have

$$
p\left(\langle\theta(x), \theta(y)\rangle-\mathbf{c}\left(b_{p}\right)\langle x, y\rangle\right) p=0 \quad(x, y \in E) .
$$

As the weak-*-closed linear span, $I$, of $\left\{\langle\theta(x), \theta(y)\rangle-\mathbf{c}\left(b_{p}\right)\langle x, y\rangle: x, y \in E\right\}$ is an ideal of $A^{* *}$, there is a central projection $q_{I} \in A^{* *}$ with $I=q_{I} A^{* *}$. Since $p q_{I}=p q_{I} p=0$, we have $\mathbf{c}(p) \leq 1-q_{I}$. Consequently,

$$
\mathbf{c}(p)\langle\theta(x), \theta(y)\rangle=\mathbf{c}(p) \mathbf{c}\left(b_{p}\right)\langle x, y\rangle \quad(x, y \in E) .
$$

Now, let $\mathcal{D}:=\{D \subseteq P: \mathbf{c}(p) \mathbf{c}(q)=0$ whenever $(x, p),(y, q) \in D\}$. If we equip $\mathcal{D}$ with the usual inclusion, then Zorn's Lemma gives a maximal element $D_{0}=$ $\left\{\left(x_{\gamma}, p_{\gamma}\right)\right\}_{\gamma \in \Gamma} \in \mathcal{D}$. Set $q_{0}:=\bigvee_{\gamma \in \Gamma} \mathbf{c}\left(p_{\gamma}\right)$ in $\operatorname{Proj}_{1}\left(A^{* *}\right)$, which is a central element. Observe that $1-q_{0}$ will not dominate a nontrivial open projection. Indeed, if $0 \neq q \leq 1-q_{0}$ is an open projection, then Lemma 2.1(b) produces an element $(y, r) \in P$ such that $r \leq q$. Therefore, $D_{0} \cup\{(y, r)\} \in \mathcal{D}$, which contradicts the maximality of $D_{0}$. We now claim that the $*$-homomorphism $\Phi: A \rightarrow q_{0} A \subseteq A^{* *}$ defined by $\Phi(a)=q_{0} a$ is injective. Suppose on the contrary that there exists $a \in A_{+}$ with $\|a\|=1$ and $\Phi(a)=0$. Take any $\epsilon \in(0,1)$, and put $q_{\epsilon}$ to be the nonzero open projection $e_{a}(\epsilon, 1]$. As $a-\epsilon q_{\epsilon} \geq 0$, we have $q_{\epsilon} q_{0}=q_{0} q_{\epsilon} q_{0} \leq q_{0} a q_{0} / \epsilon=0$. So, $q_{\epsilon} \leq 1-q_{0}$, which implies the contradiction that $q_{\epsilon}=0$.

As $x_{\gamma} \mathbf{c}\left(p_{\gamma}\right)=x_{\gamma} p_{\gamma} \mathbf{c}\left(p_{\gamma}\right)=x_{\gamma}(\gamma \in \Gamma)$, we see that $x_{\gamma}$ and $x_{\gamma^{\prime}}$ are orthogonal if $\gamma \neq \gamma^{\prime}$. We now claim that the $\mathbf{c}\left(b_{\gamma}\right)$ 's are uniformly bounded (where $b_{\gamma} \in Z\left(p_{\gamma} A p_{\gamma}\right)_{+}$is the element associated with $\left(x_{\gamma}, p_{\gamma}\right) \in P$ that satisfies (2.2)). Suppose on the contrary that there are $\mathbf{c}\left(b_{\gamma_{n}}\right)$ with $\left\|\mathbf{c}\left(b_{\gamma_{n}}\right)\right\|=\left\|b_{\gamma_{n}}\right\| \geq n^{3}(n \in \mathbb{N})$. Note that the orthogonal sum $x:=\sum_{n} \frac{x_{\gamma_{n}}}{n}$ is convergent in norm in $E$. By the orthogonality-preserving property of $\theta$, Lemma 2.1(a) as well as equality (2.1), for any $m \in \mathbb{N}$,

$$
\begin{aligned}
\langle\theta(x), \theta(x)\rangle & =\left\langle\theta\left(x_{\gamma_{m}} / m\right), \theta\left(x_{\gamma_{m}} / m\right)\right\rangle+\left\langle\theta\left(x-x_{\gamma_{m}} / m\right), \theta\left(x-x_{\gamma_{m}} / m\right)\right\rangle \\
& \geq\left\langle\theta\left(x_{\gamma_{m}} / m\right), \theta\left(x_{\gamma_{m}} / m\right)\right\rangle=\frac{\mathbf{c}\left(b_{\gamma_{m}}\right)\left\langle x_{\gamma_{m}}, x_{\gamma_{m}}\right\rangle}{m^{2}}=\frac{b_{\gamma_{m}}}{m^{2}} .
\end{aligned}
$$

As the norm of the last term goes to infinity as $n \rightarrow \infty$, we reach a contradiction.

Finally, let $d$ be the weak-*-limit in $A^{* *}$ of finite sums of the uniformly bounded mutually orthogonal elements $\mathbf{c}\left(b_{\gamma}\right)$ (see Lemma 2.1(a)). By relation (2.2) and the fact that $q_{0}$ is the weak-*-limit of finite sums of $\mathbf{c}\left(p_{\gamma}\right)$ 's, we have

$$
d q_{0}\langle x, y\rangle=q_{0}\langle\theta(x), \theta(y)\rangle \in q_{0} A \quad(x, y \in E) .
$$


Since $E$ is full, we see that $d$ induces an element $m \in Z\left(M\left(q_{0} A\right)\right)_{+}$such that $m q_{0}\langle x, y\rangle=q_{0}\langle\theta(x), \theta(y)\rangle(x, y \in E)$. Since $\Phi: A \rightarrow q_{0} A$ extends to a $*-$ isomorphism $\tilde{\Phi}: M(A) \rightarrow M\left(q_{0} A\right)$, there is $u \in Z(M(A))_{+}$such that $\tilde{\Phi}(u)=m$. This means that

$$
\Phi(u\langle x, y\rangle-\langle\theta(x), \theta(y)\rangle)=0 \quad(x, y \in E),
$$

which gives the required conclusion.

Remark 2.4. Let $A$ be a general $C^{*}$-algebra. Suppose that there exist Hilbert $A^{* *}$-modules $\tilde{E}$ and $\tilde{F}$ containing $E$ and $F$ respectively such that the Hilbert $A^{* *}$ module structures extend the corresponding Hilbert $A$-module structures and $\theta$ extends to an orthogonality-preserving $A^{* *}$-module map $\tilde{\theta}: \tilde{E} \rightarrow \tilde{F}$. Then one can use Theorem 2.3 to show that $\theta$ satisfies the conclusion of Conjecture 1.2 (since $A^{* *}$ has real rank zero). In the situation when $\theta$ is a bounded orthogonalitypreserving $A$-module map, we have tried $\tilde{E}=E^{* *}$ and $\tilde{F}=F^{* *}$ but encountered some difficulties in showing that $\theta^{* *}$ is orthogonality preserving. It was claimed in [7] that, when $\theta$ is bounded, such $\tilde{E}, \tilde{F}$ and $\tilde{\theta}$ could be found. However, instead of manipulating the difficulties in the arguments in 7, we are working on a proof for the case of general $C^{*}$-algebras, without the boundedness assumption on $\theta$ (but $\theta$ is assumed to be an $A$-module map), using completely different ideas from those in this article and in [7, [9], and [13].

\section{Orthogonality-PRESERVing $\mathbb{C}$-Linear LOCAL MAPS}

In this section, we consider ( $\mathbb{C}$-linear) local maps (see the Introduction) that preserve orthogonality. Let us first give the following useful observation.

Lemma 3.1. Let $A_{0}$ be the *-algebra generated by all the idempotents in $A$. If $\Psi: E \rightarrow F$ is a local map, then $\Psi$ is an $A_{0}$-module map.

Proof. Let $p \in A$ be an idempotent and $x \in E$. As $\Psi$ is local, one has $\Psi(x-x p) p=$ 0 . If $\left\{u_{i}\right\}$ is an approximate unit for $A$, then $(1-p) u_{i} \in A$ will strictly converge to $(1-p)$. Since $\Psi(x p)(1-p) u_{i}=0$, we have $\langle y, \Psi(x p)\rangle(1-p)=\lim \langle y, \Psi(x p)\rangle(1-$ $p) u_{i}=0(y \in F)$. This implies that $\Psi(x p)-\Psi(x p) p=\Psi(x p)(1-p)=0$. Thus, $\Psi(x) p=\Psi(x p)$, and so $\Psi(x v)=\Psi(x) v$ for any $v \in A_{0}$.

Note that if $A$ has real rank zero, then $A_{0}$ is dense in $A$. We remark however that $A_{0}$ can be $\{0\}$ (e.g. if $A=C_{0}(0,1)$ ).

Recall that $A$ is a standard $C^{*}$-algebra on a Hilbert space $H$ if $\mathcal{K}(H) \subseteq A \subseteq$ $\mathcal{L}(H)$. In this case, $A_{0}$ contains a "big enough" ideal $\mathcal{F}(H)$ of $A$, in the sense that $\mathcal{K}(H)=\overline{\mathcal{F}(H)}$ is an essential ideal. As a consequence, we can use Lemma 3.1 and Theorem 2.3 to give a self-contained proof of the following slight extension of 9 , 2.3 ] (note that the $A$-linearity is replaced by the local property).

Corollary 3.2 (cf. $9,2.3]$ ). Suppose that $A$ is a standard $C^{*}$-algebra on a Hilbert space $H$. If $\Psi: E \rightarrow F$ is local and orthogonality preserving, then there is $\lambda \in \mathbb{R}_{+}$ such that

$$
\langle\Psi(x), \Psi(y)\rangle=\lambda\langle x, y\rangle \quad(x, y \in E) .
$$


Proof. Consider $E_{0}:=E \cdot \mathcal{K}(H)$ and $F_{0}:=F \cdot \mathcal{K}(H)$ (both of them being Hilbert $\mathcal{K}(H)$-modules). Let $\left\{v_{\gamma}\right\}_{\gamma \in \Gamma}$ be an approximate unit in $\mathcal{K}(H)$ consisting of finite rank positive operators. By Lemma 3.1. $\Psi(x v)=\Psi(x) v$ for every finite rank operator $v$ and every $x \in E$. On the other hand, for any $y \in E_{0}$, there exist $a \in \mathcal{K}(H)$ and $x \in E_{0}$ with $y=x a$ (by the Cohen factorization theorem), and so

$$
\Psi(y) v_{\gamma}=\Psi\left(x a v_{\gamma}\right)=\Psi(x) a v_{\gamma} \quad(\gamma \in \Gamma),
$$

which shows that $\left\|\Psi(y) v_{\gamma}-\Psi(x) a\right\| \rightarrow 0$ (along $\gamma$ ). Define $\Phi: E_{0} \rightarrow F_{0}$ by setting $\Phi(y)$ to be the norm limit of $\Psi(y) v_{\gamma}$. As $\Phi(y)=\Psi(x) a$ as well, we see that $\Phi(y)$ does not depend on the choice of $\left\{v_{\gamma}\right\}_{\gamma \in \Gamma}$, nor on the decomposition $y=x a$. If $b \in \mathcal{K}(H)$, then

$$
\Phi(y b)=\Phi(x a b)=\Psi(x) a b=\Phi(y) b .
$$

Moreover, if $x, y \in E_{0}$ with $\langle x, y\rangle=0$, then $\langle\Psi(x), \Psi(y)\rangle=0$, which implies that $\left\langle\Psi(x) v_{\gamma}, \Psi(y) v_{\gamma^{\prime}}\right\rangle=0\left(\gamma, \gamma^{\prime} \in \Gamma\right)$, and so, $\langle\Phi(x), \Phi(y)\rangle=0$. On the other hand, since $\mathcal{K}(H)$ is simple, we see that either $E_{0}$ is a full $\mathcal{K}(H)$-module or $E_{0}=\{0\}$. By Theorem 2.3, there exists $\lambda \in Z(M(\mathcal{K}(H)))_{+}=\mathbb{R}_{+}$such that for every $x, y \in E_{0}$, one has $\langle\Phi(x), \Phi(y)\rangle=\lambda\langle x, y\rangle$ (note that one can take any $\lambda$ if $E_{0}=\{0\}$ ). Thus, for any $x, y \in E$ and $\gamma, \gamma^{\prime} \in \Gamma$,

$$
v_{\gamma}\langle\Psi(x), \Psi(y)\rangle v_{\gamma^{\prime}}=\left\langle\Phi\left(x v_{\gamma}\right), \Phi\left(y v_{\gamma^{\prime}}\right)\right\rangle=\lambda\left\langle x v_{\gamma}, y v_{\gamma^{\prime}}\right\rangle=\lambda v_{\gamma}\langle x, y\rangle v_{\gamma^{\prime}} .
$$

Consequently, if $b_{x, y}:=\langle\Psi(x), \Psi(y)\rangle-\lambda\langle x, y\rangle \in A$, then $v_{\gamma} b_{x, y} v_{\gamma^{\prime}}=0\left(\gamma, \gamma^{\prime} \in \Gamma\right)$, which shows that $b_{x, y}=0$ (as $v_{\gamma} \rightarrow 1$ in the strong operator topology).

We recall that a unital $C^{*}$-algebra $A$ is said to be properly infinite if there exists $p \in \operatorname{Proj}_{1}(A)$ such that $p \sim 1 \sim 1-p$.

Corollary 3.3. Let $A$ be a properly infinite real rank zero unital $C^{*}$-algebra. If $E$ is full and $\Psi: E \rightarrow F$ is an orthogonality-preserving local map, then there is $u \in Z(A)_{+}$such that

$$
\langle\Psi(x), \Psi(y)\rangle=u\langle x, y\rangle \quad(x, y \in E) .
$$

Proof. Let $A_{1}$ be the linear span of projections in $A$. By [15, Corollary 2.2], we see that $A=A_{1}$. Therefore, Lemma 3.1 tells us that $\Psi$ is an $A$-module map. Now, we can apply Theorem 2.3 to obtain the conclusion.

Corollary 3.4. Let $A$ be a $W^{*}$-algebra. If $E$ is full and $\Psi: E \rightarrow F$ is an orthogonality-preserving local map, then there is $u \in Z(A)_{+}$such that

$$
\langle\Psi(x), \Psi(y)\rangle=u\langle x, y\rangle \quad(x, y \in E) .
$$

Proof. By Theorem 2.3 it suffices to show that $\Psi$ is an $A$-module map. Recall that there are mutually orthogonal central projections $q_{11}, q_{21}$ and $q_{\infty}$ in $A$ summing up to 1 such that $q_{11} A$ is a finite $W^{*}$-algebra of type $I, q_{21} A$ is a finite $W^{*}$-algebra of type II, and $q_{\infty} A$ is a properly infinite $W^{*}$-algebra (see, e.g., [14, 6.1.9]). Thus, $E=E q_{11} \oplus E q_{21} \oplus E q_{\infty}$. The restriction $\left.\Psi\right|_{E q_{\infty}}$ is a $\left(q_{\infty} A\right)$-module map because of Lemma 3.1 and the fact that every element in $q_{\infty} A$ is a sum of at most five idempotents (see [17, Theorem 4]). Similarly, the restriction $\left.\Psi\right|_{E q_{21}}$ is a $\left(q_{21} A\right)$ module map since every element in $q_{21} A$ is a complex linear combination of at most twenty-four projections [8, Theorem 2]. Thus, it remains to verify the case when $A$ is a finite $W^{*}$-algebra of type $I$. 
In this case, for each $n \in \mathbb{N}$, there exist a hyperstonean space $\Omega_{n}$ (could be empty) and a projection $q_{n} \in Z(A)$ such that $\left\{q_{n}\right\}$ are orthogonal to one another, $\sum_{n} q_{n}$ weak-*-converges to 1 and $q_{n} A \cong C\left(\Omega_{n}\right) \otimes M_{n}$ (see e.g. [14, 6.7.7]). Here we use the convention that $C\left(\Omega_{n}\right)=\{0\}$ if $\Omega_{n}=\emptyset$. Let $n \in \mathbb{N}$ such that $\Omega_{n} \neq \emptyset$ and $e \in C\left(\Omega_{n}\right)$ be the identity. Pick any rank one projection $p \in M_{n}$. If $r:=e \otimes p \in \operatorname{Proj}_{1}\left(q_{n} A\right)$, then $r A r$ is isomorphic to $C\left(\Omega_{n}\right)$. By Lemma 3.1, the induced map $\Psi_{r}: E r \rightarrow F r$ is an orthogonality-preserving local map between Hilbert $r A r$-modules. Using 13 , 3.5], we see that $\Psi_{r}$ is an $r A r$-module map. In particular, for any $a \in C\left(\Omega_{n}\right)$ and $x \in E$, one has

$$
\Psi(x(a \otimes p))=\Psi_{r}(x r(a \otimes I) r)=\Psi_{r}(x r) r(a \otimes I) r=\Psi(x)(a \otimes p),
$$

where $I \in M_{n}$ is the identity. Now, let $\left\{e_{k l}\right\}_{k, l=1}^{n}$ be the matrix unit of $M_{n}$. As $\frac{e_{k l}+e_{k l}^{*}+e_{k k}+e_{l l}}{2}$ and $\frac{\mathrm{i}\left(e_{k l}-e_{k l}^{*}\right)+e_{k k}+e_{l l}}{2}$ are rank one projections, we see that $e_{k l}$ is a linear combination of rank one projections $(k, l \in\{1, \ldots, n\})$. Since any $a \in q_{n} A$ is of the form $a=\sum_{i, j=1}^{n} a_{i j} \otimes e_{i j}\left(a_{i j} \in C\left(\Omega_{n}\right)\right)$, we see that $\Psi(x a)=\Psi(x) a$ $\left(x \in E ; a \in q_{n} A\right)$. It follows that for any $x \in E$ and $a \in A$,

$\Psi(x a) \sum_{k=1}^{n} q_{k}=\Psi\left(x a \sum_{k=1}^{n} q_{k}\right)=\Psi(x)\left(a \sum_{k=1}^{n} q_{k}\right)=(\Psi(x) a) \sum_{k=1}^{n} q_{k} \quad(n \in \mathbb{N})$.

Consequently, for any $y \in F$, we have $\langle y, \Psi(x a)-\Psi(x) a\rangle \sum_{k=1}^{n} q_{k}=0$, which implies that $\langle y, \Psi(x a)-\Psi(x) a\rangle=0$, and so $\Psi(x a)=\Psi(x) a$ as required.

We end this article with a result that could be a first step towards a positive answer for Conjecture 1.2 in the case when $A$ has real rank zero (with $\theta$ not being assumed to be an $A$-module map nor bounded). This result is also interesting by itself and gives us a rough idea what kind of difficulties we will come across without the $A$-linearity.

Proposition 3.5. Let $A$ be a unital $C^{*}$-algebra of real rank zero, and $A_{0}$ be the *algebra generated by the idempotents in $A$. Suppose that there is an element $x_{0} \in E$ such that $\left\langle x_{0}, x_{0}\right\rangle=1$. If $\Psi: E \rightarrow F$ is a local map preserving orthogonality, then one can find $u \in Z(A)_{+}$as well as an $A_{0}$-submodule $E_{0} \subseteq E$ containing $x_{0}$ with $E_{0}^{\perp}=\{0\}$ such that

$$
\langle\Psi(x), \Psi(y)\rangle=u\langle x, y\rangle \quad\left(x, y \in E_{0}\right) .
$$

Proof. Define $u:=\left\langle\Psi\left(x_{0}\right), \Psi\left(x_{0}\right)\right\rangle \in A_{+}$. Note that by Lemma 3.1, $\Psi$ is an $A_{0^{-}}$ module map. Thus, $\Psi(x w)=\Psi(x) w$ for any symmetry $w \in A$ (as $w \in A_{0}$ ). Now, the argument of Proposition 2.2 tells us that $u \in Z(A)_{+}$. Let $z \in E$ such that $\left\langle x_{0}, z\right\rangle=0$ and $\langle z, z\rangle \in A_{0}$. Then $z+x_{0}$ is also orthogonal to $z-x_{0}\langle z, z\rangle$. It follows from the orthogonality-preserving property and the $A_{0}$-linearity of $\Psi$ that

$$
\langle\Psi(z), \Psi(z)\rangle=\left\langle\Psi\left(x_{0}\right), \Psi\left(x_{0}\right)\right\rangle\langle z, z\rangle=u\langle z, z\rangle .
$$

Let $\mathcal{D}:=\left\{D \subseteq E: x_{0} \in D ;\langle x, x\rangle \in A_{0}\right.$ and $\langle x, y\rangle=0$ for any $\left.x \neq y \in D\right\}$. Take any maximal element $M$ in $\mathcal{D}$, and define $E_{0}$ to be the linear spans of $x \cdot a$ $\left(x \in M ; a \in A_{0}\right)$. For any $y \in E_{0}$, we know that $\langle y, y\rangle,\left\langle x_{0}, y\right\rangle \in A_{0}$. Thus, $z=y-x_{0}\left\langle x_{0}, y\right\rangle$ is orthogonal to $x_{0}$ and $\langle z, z\rangle=\langle y, y\rangle-\left\langle y, x_{0}\right\rangle\left\langle x_{0}, y\right\rangle \in A_{0}$. Hence, the above implies that

$$
\langle\Psi(y), \Psi(y)\rangle=\left\langle y, x_{0}\right\rangle\left\langle\Psi\left(x_{0}\right), \Psi\left(x_{0}\right)\right\rangle\left\langle x_{0}, y\right\rangle+\langle\Psi(z), \Psi(z)\rangle=u\langle y, y\rangle .
$$


A polarization type argument tells us that $\langle\Psi(x), \Psi(y)\rangle=u\langle x, y\rangle\left(x, y \in E_{0}\right)$. Suppose on the contrary that there exists $z \in E$ with $\|z\|=1$ and $\langle z, x\rangle=0$ for any $x \in E_{0}$. Let $a:=\langle z, z\rangle$ and $q_{n}:=e_{a}\left(\frac{1}{2^{n}}, 1\right](n \in \mathbb{N})$. There exist $d, b \in C^{*}(a)_{+}$ such that $q_{5} \leq a b \leq 1, d \leq a, d q_{4}=a q_{4}$ and $d q_{5}=d$. As $b^{1 / 2} d^{1 / 2} \leq 1, b^{1 / 2} d^{1 / 2} q_{4}=$ $b^{1 / 2} a^{1 / 2} q_{4}=q_{4}$ and $b^{1 / 2} d^{1 / 2} q_{5}=b^{1 / 2} d^{1 / 2}$, we see that

$$
\left\|z-z b^{1 / 2} d^{1 / 2}\right\|^{2}=\left\|a-2 a b^{1 / 2} d^{1 / 2}+a b d\right\| \leq 2\left\|a\left(1-b^{1 / 2} d^{1 / 2}\right)\right\|<1 / 8 .
$$

Since $d^{1 / 2} \in \operatorname{her}\left(q_{5}\right)$, there exists $c \in A_{0} \cap \operatorname{her}\left(q_{5}\right)_{+}$such that $\left\|d^{1 / 2}-c\right\|<1 / 8$ (because her $\left(q_{5}\right)$ also has real rank zero; see e.g. [5]). If $z^{\prime}:=z b^{1 / 2} c$, then $\left\langle z^{\prime}, z^{\prime}\right\rangle=$ $c q_{5} a b q_{5} c=c^{2} \in A_{0}\left(\right.$ as $\left.a b q_{5}=q_{5}\right)$ and $\left\|z-z^{\prime}\right\| \leq\left\|z-z b^{1 / 2} d^{1 / 2}\right\|+\| z b^{1 / 2} d^{1 / 2}-$ $z b^{1 / 2} c \| \leq 1 / 2$, which implies that $z^{\prime} \neq 0$. Moreover, since $\left\langle x, z^{\prime}\right\rangle=\langle x, z\rangle b^{1 / 2} c=0$ for any $x \in M$, we see that $M \cup\left\{z^{\prime}\right\} \in \mathcal{D}$, which is a contradiction.

\section{REFERENCES}

[1] J. Alaminos, M. Brešar, M. Černe, J. Extremera, and A. R. Villena, Zero product preserving maps on $C^{1}[0,1]$, J. Math. Anal. Appl. 347 (2008), 472-481. MR2440343 (2009j:47069)

[2] J. Araujo, Linear biseparating maps between spaces of vector-valued differentiable functions and automatic continuity, Adv. Math. 187 (2004), no. 2, 488-520. MR2078345(2005c:47040)

[3] A. Blanco and A. Turnšek, On maps that preserve orthogonality in normed spaces, Proc. Royal Soc. Edinburgh 136A (2006), 709-716. MR 2250441 (2007e:46006)

[4] L. G. Brown, Semicontinuity and multipliers of $C^{*}$-algebras, Can. J. Math., vol. XL, no. 4 (1988), 865-988. MR969204 (90a:46148)

[5] L. G. Brown and G. K. Pedersen, $C^{*}$-algebras of real rank zero, J. Funct. Anal. 99 (1991), 131-149. MR:1120918 (92m:46086)

[6] J. Chmieliński, Linear mappings approximately preserving orthogonality, J. Math. Anal. Appl. 304 (2005), 158-169. MR.2124655 (2005m:46118)

[7] M. Frank, A.S. Mishchenko and A.A. Pavlov, Orthogonality-preserving, $C^{*}$-conformal and conformal module mappings on Hilbert $C^{*}$-modules, J. Funct. Anal. 260 (2011), 327-339. MR2737403

[8] S. Goldstein and A. Paszkiewicz, Linear combinations of projections in von Neumann algebras, Proc. Amer. Math. Soc. 116 (1992), 175-183. MR1094501 (92k:46101)

[9] D. Ilišević and A. Turnšek, Approximately orthogonality preserving mappings on $C^{*}$-modules, J. Math. Anal. Appl. 341 (2008), 298-308. MR2394085 (2009i:46109)

[10] R. V. Kadison and J. R. Ringrose, Fundamentals of the theory of operator algebras, Vol. I, Graduate Studies in Math, 15. Amer. Math. Soc., Providence, RI, 1997. MR 1468229 (98f:46001a)

[11] R. Kantrowitz and M. M. Neumann, Disjointness preserving and local operators on algebras of differentiable functions, Glasg. Math. J. 43 (2001), 295-309. MR.1838633 (2002d:47048)

[12] C.-W. Leung, C.-K. Ng and N.-C. Wong, Automatic continuity and $C_{0}(\Omega)$-linearity of linear maps between $C_{0}(\Omega)$-modules, J. Aust. Math. Soc. 89 (2010), 245-254.

[13] C.-W. Leung, C.-K. Ng and N.-C. Wong, Linear orthogonality preservers of Hilbert bundles, J. Aust. Math. Soc. 89 (2010), 245-254. MR2769139

[14] Bingren Li, Introduction to operator algebras, World Scientific, Singapore, 1992. MR 1194183 (94b:46083)

[15] Y.-F. Lin and M. Mathieu, Jordan isomorphism of purely infinite $C^{*}$-algebras, Quart. J. Math. 58 (2007), 249-253. MR2334865 (2008k:46189)

[16] R. Narasimhan, Analysis on real and complex manifolds, Advanced Studies in Pure Mathematics 1, North-Holland Publishing Co., Amsterdam, 1968. MR0251745 (40:4972)

[17] C. Pearcy and D. Topping, Sums of small numbers of idempotents, Michigan Math. J. 14 (1967), 453-465. MR0218922 (36:2006)

[18] G. K. Pedersen, $C^{*}$-algebras and their automorphism groups, Academic Press, New York, 1979. MR548006 (81e:46037)

[19] J. Peetre, Réctification à l'article "Une caractérisation abstraite des opérateurs différentiels", Math. Scand. 8 (1960), 116-120. MR0124611 (23:A1923) 
[20] Jürgen Schweizer, Interplay between noncommutative topology and operators on $C^{*}$-algebras, PhD thesis, Univ. Tuebingen (1996).

[21] Jürgen Schweizer, An analogue of Peetre's theorem in non-commutative topology, Q. J. Math. 52 (2001), 499-506. MR1874495(2002j:58013)

Department of Mathematics, The Chinese University of Hong Kong, Shatin, N.T., Hong Kong SAR, People's Republic of China

E-mail address: cwleung@math.cuhk.edu.hk

Chern Institute of Mathematics and LPMC, Nankai University, Tianjin 300071, PeoPLE'S REPUBlic OF CHINA

E-mail address: ckng@nankai.edu.cn

Department of Applied Mathematics, National Sun Yat-sen University, Kaohsiung, 80424, Taiwan, Republic of China

E-mail address: wong@math.nsysu.edu.tw 